\title{
Is Bacterial biobutanol production a viable solution?
}

\author{
Jules Thibault \\ Department of Chemical and Biological Engineering \\ University of Ottawa \\ Ottawa (ON) Canada K1N 6N5 \\ Jules.Thibault@uottawa.ca
}

\section{Extended Abstract}

Butanol is considered as a promising sustainable biofuel to partly replace petroleum-based liquid fuels. Biobutanol is a superior biofuel compared to bioethanol. However, to become an economically-viable biofuel, some challenges need to be overcome in the biobutanol production process such as the low final product concentration caused by product toxicity to the microorganism. Another hurdle is the relatively low yield of butanol produced by the commonly-used Clostridial bacterial strains including Clostridium acetobutylicum and Clostridium beijerinckii as other chemicals are formed, namely acetone, ethanol, butyric acid, acetic acid, hydrogen and carbon dioxide. To overcome these challenges, it is possible to search for new microorganisms or to genetically modify current biobutanol-producing microorganisms to increase the yield and final concentration of butanol. Another route is to use product-recovery technologies (vacuum fermentation, adsorption, pervaporation, gas stripping, liquid-liquid extraction, perstraction) to extract biobutanol in situ or ex situ from dilute fermentation broths in view of improving productivity.

In this presentation, an overview of the biochemical production of butanol with native and genetically-modified microorganisms will be reviewed and the various product-recovery technologies will be examined to demonstrate how these technologies can be implemented to improve the overall production process. An emphasis will be placed on methods to improve biobutanol productivity. It is the combination of biochemical strain improvements and production process integration that can lead to an economically-viable industry and could allow penetrating a larger biofuel market. 